\title{
New possibility of traditional Chinese and Japanese medicine as treatment for behavioral and psychiatric symptoms in dementia
}

This article was published in the following Dove Press journal:

Clinical Interventions in Aging

3 October 2012

Number of times this article has been viewed

\author{
Fan-Chin Kung' \\ Ryouhei Ishii ${ }^{2}$ \\ Hsing-Cheng Liu ${ }^{3}$ \\ Masatoshi Takeda ${ }^{2}$ \\ 'Yuli Hospital, DOH, Hualien, Taiwan; \\ 2Department of Psychiatry, Osaka \\ University Graduate School of \\ Medicine, Osaka, Japan; ${ }^{3}$ Department \\ of General Psychiatry, Taipei City \\ Psychiatric Center, Taipei City \\ Hospital, Taipei, Taiwan
}

\begin{abstract}
Yokukansan, one of the Kampo prescriptions, is composed of seven herbaceous plants and was developed in China in the 16th century as a cure for restlessness and agitation in children. Yokukansan has also become a popular drug combination in Japan, especially for the behavioral and psychiatric symptoms of dementia (BPSD). Recent studies have shown that yokukansan might also be quite effective against BPSD occurring in association with other types of dementia, such as Alzheimer's disease, Lewy body disease, Parkinson's disease with dementia, frontotemporal dementia, and vascular dementia. Researchers have intensively investigated yokukansan, focusing on the pharmacological mechanisms against glutamate-mediated excitotoxicity. This traditional Chinese and Japanese medicine holds potential promise for improving BPSD in elderly patients suffering from dementia.
\end{abstract}

Keywords: yokukansan, dementia, Alzheimer's disease, behavioral and psychological symptoms of dementia, Kampo, glutamate

The behavioral and psychiatric symptoms of dementia (BPSD) are a heterogeneous group of symptoms and behaviors occurring in patients with different types of dementia. In Taiwan, among several of the severe disturbances seen in patients with dementia, BPSD are frequent, as in other areas of the world. ${ }^{1-3}$ BPSD include delusions, hallucinations, affective symptoms, anxiety, sleep problems, aggressive behavior, activity disturbance, and many other behavioral problems, and occur in $20 \%-80 \%$ of patients with dementia. These symptoms are associated with poor outcomes, causing distress to patients and their caregivers, increasing hospitalizations, misuse of medication, and increased costs. ${ }^{4,5}$ Lanari et al reported that an imbalance between the different types of neurotransmitters, including acetylcholine, serotonin, dopamine, and noradrenaline, might be involved in the pathophysiology of BPSD. ${ }^{6}$ Atypical antipsychotics, often used in the treatment of BPSD, cause several adverse effects, such as cognitive dysfunction, gait disturbance, and extrapyramidal symptoms. ${ }^{7}$ In addition, the US Food and Drug Administration warned in 2005 that antipsychotic medications increase mortality among elderly patients. Therefore, safer treatment options for patients with dementia and BPSD are needed.

Taiwan and Japan have a similar emerging situation for dementia. The aging population over 65 years of age in Taiwan increased from $8.6 \%$ in 2000 to $10.2 \%$ in $2007,{ }^{8}$ and the estimated average life expectancy for men and women was 75 years and 81 years, respectively, in 2007. Age-related disorders, including Alzheimer's disease 
and other types of dementia, have now become a major public health problem in Taiwan. Several studies have reported that the prevalence of dementia in Taiwan is $1.7 \%-4.3 \%$ for adults aged over 65 years, and increases further with advancing age. ${ }^{8}$ Therefore, the need to address public health problems related to dementia is of urgent importance in this region of the world.

Since 2000, the national health insurance program in Taiwan has approved and funded acetylcholinesterase inhibitors and N-methyl d-aspartate (NMDA) receptor antagonists as pharmacological therapies for dementia. Acetylcholinesterase inhibitors, such as donepezil, rivastigmine, and galantamine, and NMDA receptor antagonists such as memantine are approved for treatment of mild, moderate, and severe Alzheimer's disease. ${ }^{8}$ Because these drugs (with the exception of donepezil) were relatively recently approved by the Japanese government (in 2011), Taiwanese doctors might have more experience and knowledge about the use of these drugs for dementia than do their counterparts in Japan. ${ }^{9}$ However, Fuh et al reported that only a small proportion of Taiwanese patients with Alzheimer's disease receive licensed medical treatment because of the strict reimbursement regulations, and that these medications can only be prescribed by neurologists and psychiatrists, with periodic update using the Mini-Mental State Examination or Clinical Dementia Rating assessments being required. They suggested that knowledge about the efficacy of drug treatment and diagnostic validity might still be limited in Taiwan. ${ }^{8}$ Therefore, we should exchange knowledge and information about these new antidementia drugs between our countries.

Traditional Chinese medicine is one of the possible solutions utilized for more than 2000 years in China and several Far East Asian countries for the treatment of elderly patients suffering from dementia, with apparent efficacy and safety. ${ }^{10}$ Yokukansan (Yi-gan-san) is one of the Kampo prescriptions, composed of seven herbaceous plants, ${ }^{11}$ and was developed in China in the 16th century as a cure for restlessness and agitation in children. Chinese herbal medicines have been modified over a long time period in Japan. ${ }^{12}$ More than 120 Kampo prescriptions have been approved by the Japanese Ministry of Health for use in clinical practice, including yokukansan. ${ }^{13}$ Modern packages of the mixed dried extract of these herbs contain $2.5 \mathrm{~g}$, which are to be taken three times daily before meals. ${ }^{14}$ In Japan, yokukansan is often used for treatment of BPSD including insomnia, irritability, hypnic myoclonia, screaming attacks, and sleep tremors. ${ }^{12,15,16}$

Previous clinical studies of yokukansan have reported its usefulness for treating BPSD in patients with
Alzheimer's disease. Iwasaki et al reported that BPSD, including aggression, agitation, and irritability improved in 52 patients with Alzheimer's disease treated with yokukansan $7.5 \mathrm{~g} /$ day for one month. ${ }^{10}$ Further, Mizukami et al reported that yokukansan was safe and effective for the treatment of dementia symptoms in 106 patients diagnosed with Alzheimer's disease. They evaluated cognitive function and BPSD using the Mini-Mental State Examination and the Neuropsychiatric Inventory (NPI), respectively. They demonstrated a significant improvement in NPI scores during the treatment period as well as in NPI subscales, including depression, anxiety, delusions, hallucinations, irritability, and agitation/aggression, which lasted for 1 month. Although there was no apparent effect of yokukansan on cognitive function, the authors reported the effectiveness and tolerability of yokukansan in patients with BPSD. ${ }^{5}$ Another clinical study reported on 15 patients with Alzheimer's disease treated with yokukansan $7.5 \mathrm{~g}$ /day for 12 weeks. ${ }^{17}$ They showed a significant improvement in NPI scores in the treatment group but no improvement in the control group. They suggested that BPSD scores in elderly patients were improved by taking yokukansan, with a reduction in the doses of antipsychotics required for treatment of BPSD without any adverse effects. Further evidence of the efficacy of yokukansan was observed in an open, randomized, controlled trial in patients with Alzheimer's disease, which reported that NPI scores improved more in patients receiving a combination of donepezil and yokukansan than in patients receiving donepezil only. ${ }^{18}$

Recent studies have shown that yokukansan might also be effective against BPSD associated with types of dementia, including Lewy body disease, Parkinson disease with dementia, frontotemporal dementia, and vascular dementia. Shinno et al reported that BPSD, including psychiatric symptoms and sleep, in patients with Lewy body disease or Alzheimer's disease were improved by yokukansan. ${ }^{19,20}$ Mizukami et al also reported that patients with Alzheimer's disease or Lewy body disease taking yokukansan showed significant reductions in delusions, hallucinations, agitation/ aggression, depression, anxiety, and irritability. ${ }^{21}$ Other researchers have also reported improvement in BPSD in patients with frontotemporal dementia or Parkinson's disease with dementia treated with yokukansan. ${ }^{22,23}$ In the most recent study, Nagata et al reported the efficacy of yokukansan in patients with vascular dementia and BPSD. Thirteen Japanese patients (nine men and four women) with vascular dementia showed significant improvement after treatment with yokukansan $7.5 \mathrm{~g}$ /day for 4 weeks without any adverse effects. 
In the NPI subcategories, there was significant improvement in agitation and disinhibition after treatment. These investigators suggested that yokukansan is beneficial for the treatment of BPSD in patients with vascular dementia. ${ }^{24}$

Medicinal chemists in Japan have also investigated yokukansan intensively, focusing on the pharmacological mechanisms involved in glutamate-mediated excitotoxicity. ${ }^{11}$ Because yokukansan contains compounds with an antagonizing affinity for serotonin and dopamine receptors, it is unlikely to have extrapyramidal side effects, as do most antipsychotic drugs. ${ }^{10}$

Tateno et al demonstrated that yokukansan inhibited $\mathrm{A} \beta$-induced cytotoxicity in a primary culture of cortical neurons in rats. ${ }^{14}$ They indicated that yokukansan provoked an anticytotoxic mechanism to protect against cell death induced by glutamate. It has also been said that yokukansan reduces glutamate release in the hippocampus of rats with zinc deficiency ${ }^{13}$ and 5-HT2A receptor expression in the prefrontal rat cortex, ${ }^{25}$ and that it increases acetylcholine levels and acetyltransferase activity in experimental animals. ${ }^{26,27}$ As suggested by Tabuchi et al, yokukansan ameliorates cognitive and noncognitive symptoms in experimental rats with Alzheimer's disease without any relationship to $\mathrm{A} \beta$ deposition. ${ }^{28}$ Kawakami et al investigated the effects of yokukansan on glutamate uptake function using cultured cortical astrocytes in a rat model. The uptake of glutamate into astrocytes and levels of glutamate aspartate transporter proteins and their mRNA expression in astrocytes significantly decreased under thiamine-deficient conditions in a dose-dependent manner during treatment with yokukansan. These researchers also demonstrated dose-dependent effects of yokukansan on glutamate-induced neuronal death, and suggested that yokukansan might have a neuroprotective effect against the excitotoxicity induced by glutamate, which might be caused not only by amelioration of astrocyte dysfunction but also by directly protecting neuronal cells. ${ }^{29}$

Taiwan's medical pluralism is characterized by a dual professional system consisting of two traditions, ie, traditional Chinese medicine and Western medicine. ${ }^{30}$ Although traditional Chinese medicine has been included in Taiwan's national health insurance since 1995, the Chinese herbal medicines covered by insurance are limited to powdered extract preparations prescribed by traditional Chinese medicine practitioners. Patients need to pay themselves for crude drugs and other traditional Chinese medicine products produced according to traditional methods, such as tea bags, decoctions, pills, ointments, and capsules. That is the reason why, in Taiwan, Chinese medicine hospitals or clinics usually encourage their patients to pay extra for traditional Chinese medicine preparations not covered by national health insurance.

Yokukansan is also widely prescribed for neurotic and depressive states and is covered by national health insurance, but not for dementia and BPSD. There are more than ten Chinese medicine companies producing yokukansan in Taiwan, and the price is quite reasonable at about 360 NTD (about 900 Japanese yen, 11 US dollars) per $200 \mathrm{~g}$ powder can. The average amount is $12 \mathrm{~g} /$ day in divided doses (Dr Tseng Kuo-Lieh, Kuo-Lieh Chinese Medical Clinic, personal communication, December 31, 2011).

As reviewed here, yokukansan has become quite popular in Japan, especially for BPSD in patients with dementia. Clinical researchers in Japan are investigating the therapeutic mechanisms and positive outcomes in these patients. We hope that psychiatrists and researchers worldwide can share our knowledge and experience about antidementia drugs and the potential use of this traditional Chinese and Japanese medicine to help elderly patients suffering from dementia and BPSD.

\section{Disclosure}

The authors report no conflicts of interest in this work.

\section{References}

1. Hwang JP, Yang CH, Tsai SJ, et al. Behavioral disturbances in psychiatric inpatients with dementia of the Alzheimer's type in Taiwan. Int J Geriatr Psychiatry. 1997;12(9):902-906.

2. Chow TW, Liu CK, Fuh JL, et al. Neuropsychiatric symptoms of Alzheimer's disease differ in Chinese and American patients. Int $J$ Geriatr Psychiatry. 2002;17(1):22-28.

3. Fuh JL, Liu CY, Wang SJ, et al. Revised memory and behavior problems checklist in Taiwanese patients with Alzheimer's disease. Int Psychogeriatr. 1999;11(2):181-189.

4. Lawlor BA. Behavioural and psychological symptoms in dementia: the role of atypical antipsychotics. J Clin Psychiatry. 2004;65(11):5-10.

5. Mizukami K, Asada T, Kinoshita T, et al. A randomized cross-over study of traditional Japanese medicine (Kampo), yokukansan, in the treatment of behavioural and psychological symptoms of dementia. Int J Neuropsychopharmacol. 2009;12(2):191-199.

6. Lanari A, Amenta F, Silvestrelli G, Tomassoni D, Parnetti L. Neurotransmitter deficits in behavioral and psychological symptoms of Alzheimer's disease. Mech Ageing Dev. 2006;127(2):158-165.

7. Efficacy and adverse effects of atypical antipsychotics for dementia: meta analysis of randomised, placebo-controlled trials. Am J Geriatr Psychiatry. 2006;14(3):191-210.

8. Fuh JL, Wang SJ. Dementia in Taiwan: past, present, and future. Acta Neurol Taiwan. 2008;17(3):153-161.

9. Takeda M. Integration of drugs and non-pharmacological intervention to Alzheimer patients. Psychogeriatrics. 2012;12(1):1-2.

10. Iwasaki K, Satoh-Nakagawa T, Maruyama M, et al. A randomized, observer-blind, controlled trial of the traditional Chinese medicine YiGan San for improvement of behavioural and psychological symptoms and activities of daily living in dementia patients. J Clin Psychiatry. 2005;66(2):248-252.

11. de Caires S, Steenkamp V. Use of yokukansan (TJ-54) in the treatment of neurological disorders: a review. Phytother Res. 2010;24(9): $1265-1270$. 
12. Okamoto H, Okami T, Ikeda M, Takeuchi T. Effects of yokukansan on undifferentiated somatoform disorder with tinnitus. Eur Psychiatry. 2004;20(1):74-75.

13. Takeda A, Itoh H, Tamano H, Yuzurihara M, Oku N. Suppressive effect of yokukansan on excessive release of glutamate and aspartate in the hippocampus of zinc-deficient rats. Nutr Neurosci. 2008;11(1):41-46.

14. Tateno M, Ukai W, Ono T, Saito S, Hashimoto E, Saito T. Neuroprotective effects of Yi-Gan San against beta amyloid-induced cytotoxicity on rat cortical neurons. Prog Neuropsychopharmacol. 2008;32(7):1704-1707.

15. Ishii K. Sleep disorders in the elderly and Kampo medicine. Kampo Newest Ther. 2000;9(1):21-26.

16. Aizawa R, Kanbayashi T, Saito Y, et al. Effects of Yoku-kansan-kachimpi-hange on the sleep of normal healthy adult subjects. Psychiatry Clin Neurosci. 2002;56(3):303-304.

17. Monji A, Takita M, Samejima T, et al. Effects of yokukansan on the behavioural and psychological symptoms of dementia in elderly patients with Alzheimer's disease. Prog Neuropsychopharmacol. 2009; 33(2):308-311.

18. Okahara K, Ishida Y, Hayashi Y, et al. Effects of yokukansan on behavioral and psychological symptoms of dementia in regular treatment of Alzheimer's disease. Prog Neuropsychopharmacol Biol Psychiatry. 2010;34(3):532-536.

19. Shinno H, Inami $Y$, Inagaki $T$, et al. Effect of yi-gan san on psychiatric symptoms and sleep structure at patients with behavioral and psychological symptoms of dementia. Prog Neuropsychopharmacol Biol Psychiatry. 2008;32(3):881-885.

20. Shinno H, Utani E, Okazaki S, et al. Successful treatment with yi-gan san for psychosis and sleep disturbance in a patient with dementia with Lewy bodies. Prog Neuropsychopharmacol Biol Psychiatry. 2007;31(7):1543-1545.

21. Mizukami KA, Asada TA, Kinoshita TB, et al. A randomized cross-over study of a traditional Japanese medicine (Kampo), yokukansan, in the treatment of the behavioural and psychological symptoms of dementia. Int J Neuropsychopharmacol. 2009;12(2):191-199.
22. Kimura T, Hayashida H, Furukawa H, et al. Five cases of frontotemporal dementia with behavioral symptoms improved by yokukansan. Psychogeriatrics. 2009;9(1):38-43.

23. Kawanabe T, Yoritaka A, Shimura H, et al. Successful treatment with yokukansan for behavioral and psychological symptoms of Parkinsonian dementia. Prog Neuropsychopharmacol Biol Psychiatry. 2010;34(2):284-287.

24. Nagata K, Yokoyama E, Yamazaki T, et al. Effects of yokukansan on behavioral and psychological symptoms of vascular dementia: an openlabel trial. Phytomedicine. 2012;19(6):524-528.

25. Egashira N, Iwasaki K, Ishibashi A, et al. Repeated administration of yokukansan inhibits DOI-induced head-twitch response and decreases expression of 5-hydroxytryptamine (5-HT) 2A receptors in the prefrontal cortex. Prog Neuropsychopharmacol Biol Psychiatry. 2008;32(6):1516-1520.

26. Ito T. Regulatory effects of yoku-kan-san-ka-chimpi-hange and saikokaryukotsu-borei-to on function of the central nervous system in mice. Prog Med. 1997;17:823-830.

27. Yabe T, Torizuka K, Yamada H. Effects of Kampo medicines on choline acetyltransferase activity in rat embryo septal cultures. J Trad Med. 1995;12:54-60.

28. Tabuchi M, Yamaguchi T, Iizuka S, Imamura S. Ameliorative effects of yokukansan, a traditional Japanese medicine, on learning and noncognitive disturbances in the Tg2576 mouse model of Alzheimer's disease. J Ethnopharmacol. 2009;122(1):157-162.

29. Kawakami Z, Kanno H, Ueki T, et al. Neuroprotective effects of yokukansan, a traditional Japanese medicine, on glutamate-mediated excitotoxicity in cultured cells. Neuroscience. 2009;159(4):1397-1407.

30. Shih SF, Lew-Ting CY, Chang HY, Kuo KN. Insurance covered and non-covered complementary and alternative medicine utilization among adults in Taiwan. Soc Sci Med. 2008;67(7):1183-1189.
Clinical Interventions in Aging

\section{Publish your work in this journal}

Clinical Interventions in Aging is an international, peer-reviewed journal focusing on evidence-based reports on the value or lack thereof of treatments intended to prevent or delay the onset of maladaptive correlates of aging in human beings. This journal is indexed on PubMed Central, MedLine, the American Chemical Society's 'Chemical Abstracts Ser-

\section{Dovepress}

vice' (CAS), Scopus and the Elsevier Bibliographic databases. The manuscript management system is completely online and includes a very quick and fair peer-review system, which is all easy to use. Visit http://www.dovepress.com/testimonials.php to read real quotes from published authors. 